\title{
Evaluation of Dyslipidaemia among Different Age Groups in Urban South Indians
}

\author{
Ramesh Kalhalli Narayanaswamy ${ }^{1}$, Wilma Delphine Silvia Chickballapur Rayappa ${ }^{2}$, Pankaja Ramesh ${ }^{3}$ \\ ${ }^{1}$ Department of General Medicine, Fortis Hospital, Cunningham Road, Bangalore and Bowring and Lady \\ Curzon Medical College \& Research Institute, Bangalore, Karnataka, India. ${ }^{2}$ Department of Biochemistry, \\ Bowring and Lady Curzon Medical College \& Research Institute, Bangalore, Karnataka, India. ${ }^{3}$ Department \\ of General Medicine, Navachethana Hospital, Bangalore, Karnataka, India.
}

\section{ABSTRACT}

\section{BACKGROUND}

The significant reason for morbidity and mortality in industrialised countries is coronary artery disease. Globally, it is considered as one of the major public health problems. There is emerging evidence of premature coronary artery disease occurring in Asian Indians at least 10 years earlier as compared to other ethnic groups. The most important atherosclerotic risk factor is dyslipidaemia. Recent studies have reported that Total Cholesterol (TC), Low Density Lipoproteins (LDL), Triglycerides (TG) levels have increased among young urban population over a 20year period. Hence, this study was carried out to find out the prevalence of dyslipidaemia among different age groups in urban South Indian population.

\section{METHODS}

This study is a descriptive cross-sectional study conducted among patients visiting the OPD of Department of General Medicine. The study subjects were divided into 3 groups as per age as 18 - 40 years, $41-60$ years and $>60$ years by involving 1989 subjects of whom 532 were in 18 - 40 years age group, 522 were in 41 - 60 years age group and 935 were over 60 years of age. In this study, fasting serum lipid profile was estimated.

\section{RESULTS}

The study revealed higher prevalence of dyslipidaemia in the group $<60$ years. Statistically significant difference in mean was found with cholesterol, LDL, and triglycerides in the age group of 18 - 40 years and more than 60 years. Statistically significant difference was not found between $18-40$ years and $40-60$ years for HDL. Mean serum cholesterol was high in the age group 18 - 40 years. The highest mean triglycerides were found in the age group of 41 - 60 years. The highest mean HDL levels were found in the above 60 years age group.

\section{CONCLUSION}

Higher percentage of dyslipidaemia was found in the age groups less than 60 years. It is also the need of the hour to devise comprehensive strategy for awareness programme for periodically screening of all age groups.

\section{KEY WORDS}

Dyslipidaemia, Cholesterol, Low Density Lipoproteins, Cholesterol \& Triglyceride
Corresponding Author:

Dr. Ramesh Kalhalli Narayanaswamy. Consultant, No 14, Fortis Hospital, Cunningham Road Near Sigma Central Mall, Vasanth Nagar, Bangalore -560052, India.

E-mail: rameshknbowring@gmail.com

DOI: 10.14260/jemds/2020/655

How to Cite This Article: Narayanaswamy RK, Rayappa WDSC, Ramesh P. Evaluation of dyslipidaemia among different age groups in urban south Indians. J Evolution Med Dent Sci 2020;9(40):2992-2996, DOI: $10.14260 /$ jemds/2020/655

Submission 20-06-2020, Peer Review 27-08-2020, Acceptance 05-09-2020, Published 05-10-2020.

Copyright (C) 2020 Ramesh Kalhalli Narayanaswamy, et al. This is an open access article distributed under Creative Commons Attribution License [Attribution 4.0 International (CC BY 4.0)] 


\section{BACKGROUND}

An established risk factor for cardiovascular diseases is the elevated blood lipid level. Globally, $4.5 \%$ of total deaths are due to high total cholesterol levels. ${ }^{1-4}$ The major contributing determinant in the development of ischemic heart diseases, stroke and other vascular diseases is dyslipidaemia. According to National Cholesterol Education Programme (NCEP) dyslipidaemia is defined as hypercholesterolemia (serum cholesterol > $200 \mathrm{mg} / \mathrm{dL}$ ), hypertriglyceridemia (serum triglyceride $>150 \mathrm{mg} / \mathrm{dL}$ ) and high LDL cholesterol (LDL cholesterol $>130 \mathrm{mg} / \mathrm{dL}$ ). Studies have documented that by 2020, India will bear burden of $60 \%$ of the world's cardiovascular disease. ${ }^{5}$

A distinctive pattern of dyslipidaemia with lower HDLcholesterol, increased TG levels and higher proportion of small dense LDL-C are seen in India and there are no large population studies to evaluate the magnitude of dyslipidaemia problem in India. The prevalence of Coronary Heart Disease (CHD) is found to be around $3-4 \%$ in rural areas and a sixfold rise among the adults above 20 years of age found between the years 1960 and 2000 in urban areas. Adverse change in the lipid profile associated with urbanization associated with lifestyle with increased saturated fat consumption and decreased physical activity. The pathophysiology \& key independent amendable risk factor for cardiovascular disease is closely linked to dyslipidaemia ${ }^{6,7,8}$

The detection, evaluation and treatment guidelines for high blood cholesterol in adults was developed by National Cholesterol Education Programme (NCEP). Cardiovascular morbidity and mortality get reduced due to effective control of blood lipid levels. Hence, understanding of the various aspects of the lipid profile and the significance of each of the parameters is very essential part of the management of CHD and who are at risk of CHD. ${ }^{9,10}$ Appropriate planning of health care resources for prevention of both primary and secondary cardiovascular diseases can be ensured by estimation of prevalence of dyslipidaemia. 6

The study was planned to find out the lipid profile patterns and prevalence of dyslipidaemia in South Indian urban population between the age groups 18 to more than 60 years.

\section{METHODS}

This is a descriptive cross-sectional study conducted on 1989 patients who visited the outpatient department of General Medicine as study subjects. Study subjects were categorised into three groups: Group I: Consisting of 18 to 40 years of age, Group II: 41-60 years of age and Group III: More than 60 years of age. Subjects included in this study were more than or equal to 18 years of age of either sex., patients who volunteered to participate in this study. Subjects excluded were adults with known history of coronary heart diseases / terminally ill patients / suffering with acute or chronic illness / patients with less than 18 years of age / on medication with hypolipidemic drugs.

With aseptic precautions the blood samples were collected in the morning from the median cubital vein after a fasting period of 10 - 12 hours for lipid profile assay into dry tubes (BD Vacutainer). Informed consent was obtained from the study subjects as part of health screening before the collection of the blood sample. Ethical clearance was obtained from the Institutional ethics committee prior to this study.

Estimation of fasting lipid profile: After an overnight fasting, $5 \mathrm{ml}$ of venous blood was drawn after taking aseptic precautionary measures and allowed to clot at room temperature. Centrifugation was carried out at $3000 \mathrm{rpm}$ for 10 minutes. For separation of serum from the blood. The serum was analysed for total cholesterol by CHOD-POD (end point), triglycerides by enzymatic (GPO-PAP), LDL cholesterol \& HDL Cholesterol by Homogenous Method and Direct Measurement by using autoanalyzer within one hour of collection.

\section{Statistical Analysis}

Statistical analysis was performed with SPSS software version 22. Mean, standard deviation, and standard error were determined for each study group. Univariate analysis was carried out using Pearson Chi-square test and comparison of differences in mean in these groups was analyzed using students t-test and calculated. P-value of $<0.05$ was considered as statistically significant $t$ - test.

\section{RESULTS}

In this study, fasting lipid profile was estimated among different age groups in the urban population of South India. 1989 patients were endorsed to participate in the study as subjects. The subjects were categorised into 3 groups. Group 1 consisted of study participants in the age group of $18-40$ years, Group 2 consisted of study participants in the age group of 41 - 60 years and Group 3 consisted of study participants in the age group of more than 60 years. Out of which 532 were 18 to 40 year of age, 522 were between 41 - 60 year of age and 935 were over 60 years. The sociodemographic profile of the study subjects was collected. $70 \%$ of the study subjects were found to be male. Fasting lipid profile of all study subjects included total cholesterol, triglycerides, LDL cholesterol \& HDL cholesterol.

\begin{tabular}{|lcccc|}
\hline Lipid Profile & Age Group & N & Mean \pm SD & P Value \\
Total Cholesterol & 1 & 532 & $181.48 \pm 41.31$ & \\
& 3 & 520 & $153.81 \pm 40.2$ & 0.00 \\
LDL Cholesterol & 1 & 532 & $116.45 \pm 34.6$ & \\
& 3 & 522 & $89.53 \pm 13.3$ & 0.00 \\
Triglyceride & 1 & 532 & $145.85 \pm 32.3$ & \\
& 3 & 521 & $123.36 \pm 15.2$ & 0.00 \\
HDL Cholesterol & 1 & 532 & $36.85 \pm 9.5$ & 0.123 \\
\hline \multicolumn{5}{|c|}{ Table 1. Differences in Lipid Profile in Various Age Groups } \\
\hline \multicolumn{4}{|l}{ P' value $\leq$ 0.05 is statistically significant. } \\
\hline \multicolumn{5}{|l}{ Mean Group-1 age less than 40 years \& Group-3 age more than 60 years } \\
\hline
\end{tabular}

Tabulated the results of all 3 groups, with cholesterol $<$ $200 \mathrm{mg} / \mathrm{dL}$ and $>200 \mathrm{mg} / \mathrm{dL}, \mathrm{HDL}>45 \mathrm{mg} / \mathrm{dL}$ and $<45 \mathrm{mg}$ / dL, triglycerides $<150 \mathrm{mg} / \mathrm{dL}$ and $>150 \mathrm{mg} / \mathrm{dL}, \mathrm{LDL}<100$ $\mathrm{mg} / \mathrm{dL}$ and $>100 \mathrm{mg} / \mathrm{dL}$. Statistically significant difference was found in mean cholesterol, LDL cholesterol and triglycerides between age groups of $<40$ and $>60$ years. No statistically significant difference found between $<40$-year age group and 41 to 60 years age group (Table 1). Among the 3 
groups highest mean serum total cholesterol was observed in age group of 18 to 40 years. Mean HDL was highest in $>60$ yrs. age group, highest mean triglyceride level 147.21 was found between 41 - 60 years age group and mean LDL were high in 18 to 40 years age group as indicated in Fig 1 to 4 .
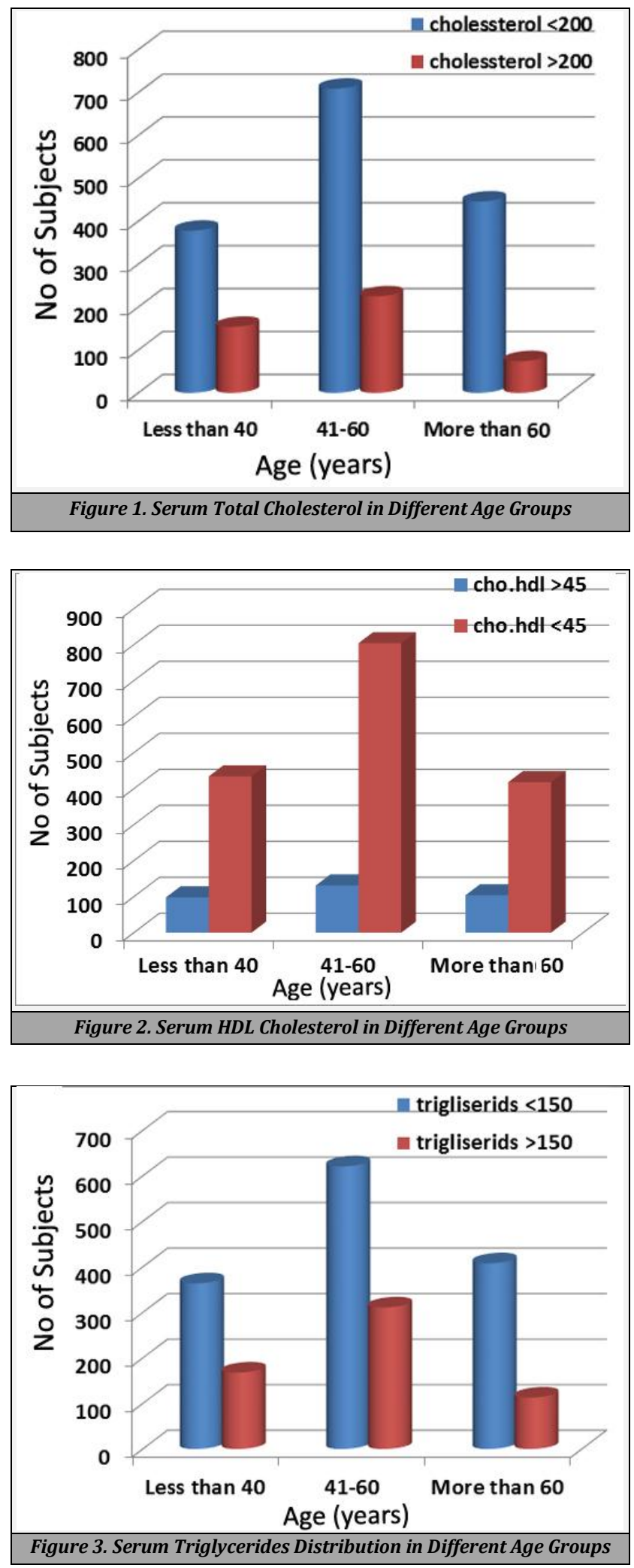

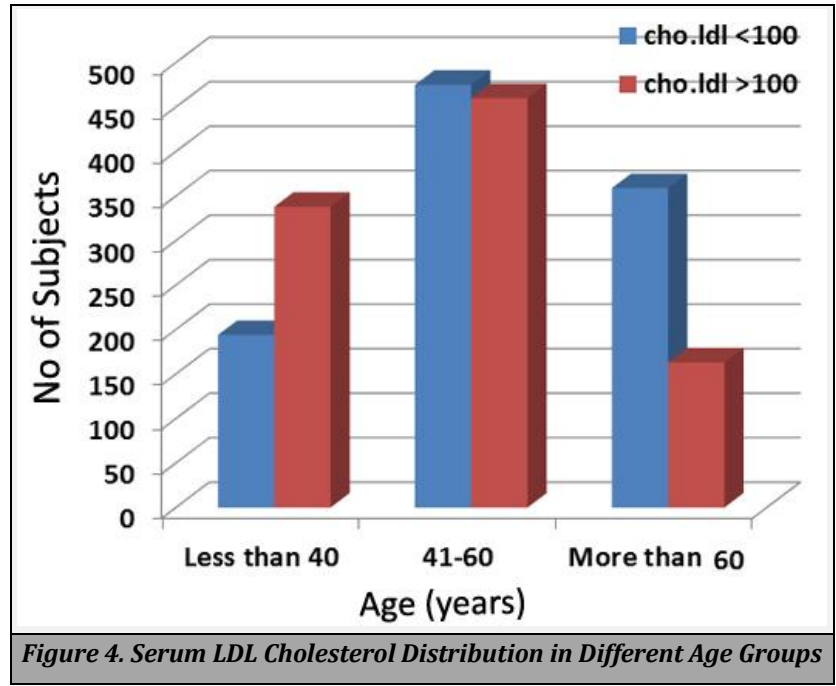

\section{DISCUSSION}

Cardiovascular risk assessment is done by lipid profile evaluation by estimating the biochemical parameters such as serum total cholesterol (TC), triglycerides (TG), low density lipoprotein-cholesterol (LDL-C) and high-density lipoprotein cholesterol (HDL-C). Evidences indicate that increased concentration of serum total cholesterol (TC), triglycerides (TG), low density lipoproteins-cholesterol (LDL-C) and decreased high density lipoprotein cholesterol (HDL-C) accelerate the development of atherosclerotic plaque. ${ }^{11,12,13}$

In this study the prevalence of hypercholesterolemia $(>$ $200 \mathrm{mg} / \mathrm{dL}$ ), high LDL ( $>130 \mathrm{mg} / \mathrm{dL}$ ) were observed in the age groups of $<40$ years and hypertriglyceridemia $(>150 \mathrm{mg}$ / dL) was observed between the age groups of 41 - 60 years. Lower HDL $<40 \mathrm{mg} / \mathrm{dL}$ was observed in $<60$ years age group.

Cross sectional studies have indicated that total cholesterol and LDL-cholesterol levels tend to elevate with age in young age and middle-aged adults and decrease in participant's $\geq 65$ years of age. However, in most of the cross sectional, prospective studies HDL cholesterol levels do not vary with age in both men and women, $14,15,16,17,18,19$ which is in accordance to this study, which showed linear association between the extent of dyslipidaemia and lesser age. There was no statically significant difference found between the prevalence of dyslipidaemia among males and females. The predominance of dyslipidaemia was high in 18 to 40 years age group.

This cross-sectional study results are concordant with the prospective study of Ferrara A et al., ${ }^{20}$ which showed statistically significant reductions in serum total cholesterol, LDL cholesterol and HDL cholesterol among males and females. The reduced capacity for the removal of LDL by the liver, probably due to reduced hepatic LDL receptor expression and / or increased intestinal absorption of cholesterol is seen with increase in LDL with age. Mixed results are found according to previous studies.

For example, significant negative correlation found between Total and LDL cholesterol and age in Honolulu Heart Program study conducted in elderly Japanese American men but in the case of Framingham Offspring study they showed positive correlation between Total and LDL cholesterol and 
age. The variation in the results of these studies may be due to the variation in the age groups inclusion criteria. The age group was between 71 and 93 yrs. in the Honolulu Heart Program while the age group was below 60 years in case of Framingham Offspring study. ${ }^{21,22,23}$ The hypothesis of increase in cholesterol levels with increase in age until the sixties but starts to decrease beyond that age supported by the findings of the present study, which indicated the tendency of decrease in both total cholesterol and LDL cholesterol with age. ${ }^{24}$ Many studies were conducted in nineties but in this study more than 60 years age group were involved to evaluate the pattern of change in lipid profile in elderly people and to evaluate if these changes would be the risk factors for coronary heart diseases. ${ }^{25}$

Recent report of cross-sectional study from Cardiovascular Health study found that HDL cholesterol levels were increased in men not in women, but most of the cross-sectional studies have not found HDL cholesterol levels varying with age. Which is similar to the results of this study which indicated increased HDL cholesterol in more than 60 years age group compared to other age groups.

Statistically significant elevation of mean serum total cholesterol was found in urban than rural participants, though there was higher concentrations of Mean serum LDL Cholesterol and Triglycerides, the difference was not statistically significant, and the most frequent dyslipidaemia found was abnormally low HDL cholesterol in the urban participants when compared to rural participants. A study was conducted by Sabir et al. to find out the plasma lipid profile levels in rural urban difference and prevalence of dyslipidaemia in Huasa Fulani of North Western Nigeria. ${ }^{26}$

Consumption patterns, smoking status, stress levels, physical activity are the risk factors for dyslipidaemia. Consumption of cholesterol in the diet increases the blood levels of cholesterol depending on the body's ability to compensate by synthesizing less cholesterol. While triglycerides in the body are influenced by carbohydrate and obesity, free radicals also cause the disruption of lipid profile by increasing the lipid peroxidation of LDL leading to formation of atheromatous plaque. ${ }^{27}$

Dyslipidaemia being a considerable and adaptable risk factor, early screening and detection and treatment can prevent the risk of Coronary artery disease among younger population.

\section{Limitations}

An observational and cross-sectional in nature of the study was primary limitation of this study. Other age groups with less than 18 years also should have been considered for the study instead of 18 to more than 60 years. Unable to document an association of physical activity, diet and other potential factors on blood lipoprotein levels in the study subjects. Future extensive studies with more potential factors and larger participant groups are required to confirm our results.

\section{CONCLUSIONS}

Lifestyle factors which are very important, and which affect the serum lipid profile include composition of diet, body weight and physical activity. Thus, crusade to improve the lifestyle factors, balanced diet, with efforts to increase physical activity may be essential to effectively reduce the cardiovascular disease risk and screening of all age groups including young population can prevent development of CAD. Early detection and interventions will help in preventing morbidity and mortality due to CAD.

Financial or Other Competing Interests: None.

\section{REFERENCES}

[1] Groot RD, den Hurk KV, Schoonmade LJ, et al. Urban-rural differences in the association between blood lipids and characteristics of the built environment: a systematic review and meta-analysis. BMJ Glob Health 2019;4(1):e001017.

[2] Wilson PW, D'Agostino RB, Levy D, et al. Prediction of coronary heart disease using risk factor categories. Circulation 1998;97(18):1837-47.

[3] Lopez AD, Mathers CD, Ezzati M, et al. Global and regional burden of disease and risk factors, 2001: systematic analysis of population health data. Lancet 2006;367(9524):1747-57.

[4] WHO. Global health risks: mortality and burden of disease attributable to selected major risks. Geneva: WHO 2009.

[5] NCEP guidelines. https://www.nhlbi.nih.gov/files/docs/guidelines/atglan ce.pdf

[6] Joshi SR, Anjana RM, Deepa M, et al. Prevalence of dyslipidaemia in urban and rural India: the ICMR-INDIAB study. PLoS ONE 2014;9(5):e96808.

[7] Groundy SM. Small LDL, atherogenic dyslipidaemia and the metabolic syndrome. Circulation 1997;95(1):1-4.

[8] Haffnar SM. Diabetes, hyperlipidemia and coronary artery disease. Am J Cardiol 1999;83(9B):17F-21F.

[9] Raj AS, Sivakumar K, Sujatha K. Prevalence of dyslipidaemia in South Indian adults: an urban-rural comparison. Int J Community Med Public Health 2016;3(8):2201-10.

[10] Pongchaiyakul C, Hongsprabhas P, Pisprasert V, et al. Rural-urban difference in lipid levels and prevalence of dyslipidaemia: a population-based study in Khon Kaen province, Thailand. J Med Assoc Thai 2006; 89(11):183544.

[11] Wei Y, Qi B, Xu J, et al. Age- and sex- related difference in lipid profiles of patients hospitalized with acute myocardial infarction in East China. J Clin Lipidol 2014;8(6):562-7.

[12] Perk J, Backer GD, Gohlke H, et al. Developed with the special contribution of the European Association for Cardiovascular Prevention \& Rehabilitation (EACPR). European guidelines on cardiovascular disease prevention in clinical practice (Version 2012). Int J Behav Med 2012;19:403-88.

[13] Gyárfás I, Keltai M, Yusuf S, et al. Effect of potentially modifiable risk factors associated with myocardial infarction in 52 countries in a case-control study based on the INTERHEART study. Orv Hetil 2006;147(15):675-86.

[14] Heiss G, Tamir I, Davis CE, et al. Lipoprotein- cholesterol distributions in selected North American populations: the 
lipid research clinics program prevalence study. Circulation 1980;61(2):302-15.

[15] Moulopoulos SD, Adamopoulos PN, Diamantopoulos EI, et al. Coronary heart disease risk factors in a random sample of athenian adults. The athens study. Am J Epidemiol 1987;126(5):882-92.

[16] Plasma lipid distributions in selected North American populations: the lipid research clinics program prevalence study. The Lipid Research Clinics Program Epidemiology Committee. Circulation 1979;60(2):42739.

[17] Abbott RD, Garrison RJ, Wilson PW, et al. Joint distribution of lipoprotein cholesterol classes. The Framingham Study. Arteriosclerosis 1983;3(3):260-72.

[18] Clark DA, Allen MF, Wilson FH. Longitudinal study of serum lipids. 12-year report. Am J Clin Nutr 1967;20(7);743-52.

[19] Hershcopf RJ, Elahi D, Andres R, et al. Longitudinal changes in serum cholesterol in man: an epidemiologic search for an etiology. J Chronic Dis 1982;35(2):101-14.

[20] Ferrara A, Barrett - Connor E, Shan J. Total, LDL and HDL cholesterol decrease with age in older men and women. The rancho bernardo study 1984-1994. Circulation 1997;96(1):37-43.
[21] Ettinger WH, Wahl PW, Kuller LH, et al. Lipoprotein lipids in older people. Results from the cardiovascular health study. The CHS collaborative research group. Circulation 1992;86(3):858-69.

[22] Ericsson S, Eriksson M, Vitols S, et al. Influence of age on the metabolism of plasma low density lipoproteins in healthy males. J Clin Invest 1991;87(2):591-6.

[23] Hollander D, Morgan D. Increase in cholesterol intestinal absorption with aging in the rat. Exp Gerontol 1979;14(4):201-4.

[24] Marhoum TA, Abdrabo AA, Lutfi MF. Effects of age and gender on serum lipid profile in over 55 years-old apparently healthy Sudanese individuals. Asian J Biomed Pharmaceutical Sci 2013;3(19):10-4.

[25] Frishman WH, Ooi WL, Derman MP, et al. Serum lipids and lipoproteins in advanced age. Intraindividual changes. Ann Epidemiol 1992;2(1-2):43-50.

[26] Sabir AA, Isezuo SA, Ohwovoriole AE, et al. Rural-urban difference in plasma lipid levels and prevalence of dyslipidaemia in hausa-fulani of North-Western Nigeria. Ethn Dis Summer. 2013;23(3):374-8.

[27] Barnoya J, Glantz SA. Cardiovascular effects of secondhand smoke: nearly as large as smoking. Circulation 2005;111(20):2684-98. 